\title{
Enfermedad tiroidea autoinmunitaria y SARS-CoV-2/COVID-19
}

\section{Autoimmune thyroid disease and SARS-CoV-2/COVID-19}

\author{
Marín $A^{1}$, Rojas $L J^{2}$, Mejía $M G^{3}$, Builes $C A^{4}$, Arenas $H M^{5}$, Duque $J^{6}$
}

${ }^{1}$ Médico Internista y Endocrinólogo. Director Médico, Endoeje.

Docente, Universidad Tecnológica de Pereira, Pereira, Risaralda.

${ }^{2}$ Médico Internista y Endocrinólogo. Máster en ciencias en Oncología

Molecular. Docente, Hospital Universitario San Ignacio, Bogotá,

Colombia.

${ }^{3}$ Médico Internista y Endocrinólogo. Máster en ciencias en Nutrición y Dietética aplicada. Docente, Hospital San José. Fundación Santafé

Bogotá, Bogotá, Colombia.

${ }^{4}$ Médico Internista y Endocrinólogo. Docente, Universidad de

Antioquia. Hospital Universitario San Vicente Fundación, Medellín,

Antioquia.

${ }^{5}$ Médico Endocrinólogo. Docente, Universidad Tecnológica de Pereira, Pereira, Risaralda.

${ }^{6}$ Médico Endocrinólogo, Clínica Central del Quindio, Armenia, Quindio.

Autor de correspondencia: Alejandro Marín

Correo electrónico: lexmarcorp@gmail.com

\section{Resumen}

Una de las preocupaciones en la pandemia de la COVID-19 es si las enfermedades tiroideas autoinmunitarias (ETA) facilitan la infección y la gravedad de las complicaciones y si el virus por sí mismo puede alterar la función tiroidea.

A la fecha, no se ha demostrado que la COVID-19 sea más frecuente o grave en pacientes con ETA; particularmente, no existe correlación con la enfermedad pulmonar grave en la COVID-19. Esto incluye a los pacientes críticamente enfermos en la unidad de cuidados intensivos (UCI). En dichos casos puede presentarse un cuadro clínico de síndrome de enfermedad no tiroidea.

Es importante que, durante el tiempo de la pandemia, se estimule la continuación del tratamiento tiroideo recibido y del control adecuado y no se suspenda durante la hospitalización.

Palabras clave: COVID-19, SARS-CoV-2, enfermedad tiroidea autoinmunitaria, tiroiditis de Hashimoto, enfermedad de Grave, pandemia, críticamente enfermo.

\section{Abstract}

One of the concerns in the face of the COVID-19 pandemic is whether autoimmune thyroid disorders facilitate infection and the severity of complications; and if the virus by itself can generate alteration in the thyroid function.

To date, there is no demonstration that COVID-19 is more frequent or severe in patients with ATD; especially, there is no correlation with severe lung disease in COVID-19. This includes the critically ill ICU patient. In such a case, the clinical picture of non-thyroid disease syndrome may occur.

It is important that the continuation of the thyroid treatment received and adequate control during the time of the pandemic be stimulated and not suspended during hospitalization.

Keywords: COVID-19, SARS-CoV-2, Autoimmune thyroid disease, Hashimoto thyroiditis, Grave's disease, Pandemic, Critically ill.

\section{Introducción}

Una de las preocupaciones ante la pandemia de la COVID-19 es si los trastornos tiroideos de tipo autoinmunitario facilitan la infección y la gravedad de las complicaciones; y si el virus por sí mismo puede generar alteración en la función tiroidea. Adicionalmente, se ha planteado una posibilidad de correlación entre los trastornos tiroideos, la disfunción pulmonar y el riesgo cardiovascular, con impacto en procesos como la disnea, el derrame pleural, la apnea del sueño y otras condiciones favorecedoras de un mayor compromiso fisiopatológico cardiorrespiratorio.

\section{Definición y epidemiología}

La enfermedad tiroidea es frecuente entre la población general. La incidencia de hipotiroidismo es más alta en mujeres que en hombres y varía según los diferentes países, de 350/100.000/año en mujeres a 80/100.000/año en hombres, siendo el hipertiroidismo primario mucho menos frecuente:

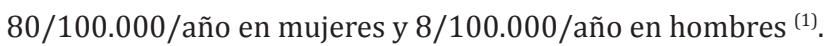




\section{Mecanismos autoinmunitarios e infecciones en enfermedad tiroidea autoinmunitaria}

La inmunidad tiroidea implica interacciones complejas entre los factores ambientales y genéticos, y se caracteriza por la reactividad de los antígenos antitiroideos y las respuestas inflamatorias o antirreceptores. En la Tabla 1 se describen las características de los anticuerpos antitiroideos: los antirreceptores de TSH, los antiperoxidasa tiroidea y los antitiroglobulina ${ }^{(2)}$.

\section{Predisposición genética}

Existe evidencia importante de concordancia de ETA en gemelos monocigóticos entre el $20 \%$ y el $40 \%$. Estas asociaciones se dan a través de ciertos alelos, como el antígeno-linfocito $\mathrm{T}$ citotóxico/proteína asociada 4, alelo G del gen CTLA-4, alelos del HLA del cromosoma 6, HLA-DRB1*08, DRB3*0202, los cuales se asocian con desarrollo de la enfermedad, y el DRB1*07 como protector ${ }^{(3)}$.

En cuanto a los genes asociados a la susceptibilidad para enfermedad tiroidea autoinmunitaria (ETA), además de HLADR, están los genes no-MHC, incluidos CTLA-4, CD40, PTPN22, tiroglobulina y los genes del receptor de TSH. Los disparadores ambientales, además del iodo, incluyen medicamentos, ta- baquismo, estrés, predisposición genética y, en algunos casos, procesos infecciosos ${ }^{(3)}$

La rubéola congénita se ha asociado con ETA, que puede iniciar la expresión de moléculas de clase II. La infección por hepatitis C es un muy conocido precipitante de ETA, cuando se asocia a la terapia con interferón (IFN), pero no se cuenta con evidencia de que esta pueda generar infección directamente ${ }^{(3,4)}$.

Se ha demostrado compromiso bacteriano por estafilococos, estreptococos, enterobacterias, bacilos Gramnegativos (como la Yersinia), anaerobios y otros menos frecuentes, como sífilis, micobacterias, hongos, como Aspergillus, o parasitarios, como en la equinococosis ${ }^{(5)}$.

Esta noxa puede inducir la expresión de las moléculas de clase II en lascélulas tiroideas humanas, y dichas células actúan como presentadoras de antígenos para iniciar una respuesta inmunitaria ${ }^{(6)}$.

Dicho fenómeno se asocia con otros procesos, como la expresión de la molécula coestimuladora de células T, CD40, en las células epiteliales tiroideas. Como aspecto adicional, las células dendríticas intratiroideas y las células B pueden servir como potentes presentadoras de antígenos. La mayoría de los antígenos principales, como TG, peroxidasa tiroidea y TSHR, que llevan a la selección de células T en los pacientes con ETA, son más frecuentemente tiroideo-específicos ${ }^{(7)}$.

Tabla 1. Caracterización de los anticuerpos antitiroideos

\begin{tabular}{|c|c|c|c|}
\hline \multicolumn{4}{|c|}{ Anticuerpos antitiroideos } \\
\hline & Anti-TSHR & Anti-TPO & Anti-Tg \\
\hline Localización del antígeno & Extracelular & Intracelular & $\begin{array}{l}\text { Folicular, bajos niveles en } \\
\text { sangre }\end{array}$ \\
\hline $\begin{array}{l}\text { Acceso de las células } \\
\text { inmunitarias al antígeno }\end{array}$ & Sin destrucción tisular & Después de destrucción tiroidea & Con y sin destrucción tisular \\
\hline $\begin{array}{l}\text { Duración de la exposición al } \\
\text { antígeno }\end{array}$ & $\begin{array}{l}\text { Corta, bajos niveles } \\
\text { normalizan pos-TTO }\end{array}$ & $\begin{array}{l}\text { Tiempo prolongado, nivel } \\
\text { intermedio }\end{array}$ & $\begin{array}{l}\text { Tiempo prolongado, niveles } \\
\text { altos }\end{array}$ \\
\hline Tipo de anticuerpo & $\begin{array}{l}\text { Oligoclonal, diferentes } \\
\text { epítopes }\end{array}$ & $\begin{array}{l}\text { Policlonal, un dominio } \\
\text { inmunodominante }\end{array}$ & $\begin{array}{l}\text { Policlonal, diferentes } \\
\text { epítopes }\end{array}$ \\
\hline Clase de anticuerpo & $\operatorname{lgG} 1$ & $\begin{array}{l}\lg G 1, \lg G 4>\lg G 2 \\
\lg G 3, \text { bajos niveles } \lg A\end{array}$ & $\begin{array}{l}\lg G 1, \lg G 4>\lg G 2 \\
\lg G 3, \text { bajos niveles } \lg A \text { e } \lg M\end{array}$ \\
\hline Prevalencia en ETA & $90 \% \mathrm{EG}, 10 \% \mathrm{TH}$ & $>80 \%$ EG y $\mathrm{TH}$ & $>50 \%$ EG y $\mathrm{TH}$ \\
\hline $\begin{array}{l}\text { Prevalencia en otras } \\
\text { enfermedades autoinmunitarias }\end{array}$ & $\begin{array}{l}\text { No expresión, } \\
18 \% \text { en DM tipo } 1\end{array}$ & $\begin{array}{l}16 \% \text { a } 37 \% \text { AR, } 40 \% \text { DM tipo } 1, \\
12 \% \text { a } 30 \% \text { EC }\end{array}$ & $\begin{array}{l}12 \% \text { a } 23 \% \text { AR, } 30 \% \text { DM tipo } \\
1,11 \% \text { a } 32 \% \text { EC }\end{array}$ \\
\hline Acción de anticuerpos & $\begin{array}{l}\text { Estimulante, bloqueante, } \\
\text { apoptosis }\end{array}$ & Poca acción per se & No acción definida \\
\hline Blancos extratiroideos & $\begin{array}{l}\text { Pocos, efectos definidos en } \\
\text { OG, DG }\end{array}$ & $\begin{array}{l}\text { Varios, acciones definidas, EH, } \\
\text { cáncer de mama }\end{array}$ & No blancos especificados \\
\hline
\end{tabular}

Anti-Tg: anticuerpos antitiroglobulina; AR: artritis reumatoidea; DG: dermopatía de Graves; DM: diabetes mellitus; EC: enfermedad celíaca; EG: enfermedad de Graves; ETA: enfermedad tiroidea autoinmunitaria; Ig: inmunoglobulina; OG: oftalmopatía de Graves; TH: tiroiditis de Hashimoto; TPO: anticuerpos antiperoxidasa tiroidea; TSHR: receptor de TSH; TTO: tratamiento.

Adaptada y traducida de la referencia 2. 


\section{Activación por efecto espectador (bystander)}

Una lesión local genera la expresión y presentación de antígenos por las moléculas de clase II ${ }^{3,8,9)}$. La tiroides responde con el cuadro de tiroiditis a la invasión por diferentes tipos virales y no a un único agente asociado. En las tiroiditis subagudas, definidas inicialmente por De Quervain, y de frecuente presentación posviral, los pacientes deben tener una susceptibilidad adicional para presentar la ETA crónica ${ }^{(10)}$. Se ha planteado la posibilidad de asociación con el antígeno HLA-B35 en algunos grupos étnicos, el cual tendría asociación con una presentación estacional (verano y otoño) ${ }^{(11)}$. También se ha descrito compromiso por virus Coxsackie, virus de Epstein-Barr (VEB), parotiditis, sarampión, enterovirus, influenza, adenovirus, espumaretrovirus (foamy retrovirus, HFV), entre otros ${ }^{(4,9)}$.

En el caso específico de las tiroiditis de Hashimoto, son también múltiples los posibles factores asociados desde el punto de vista de la infección y de predisposición involucrados, descritos a continuación.

\section{Susceptibilidad genética}

Existen asociaciones familiares. El riesgo de recurrencia en hermanos es $>20$ y la tasa de concordancia en gemelos monocigóticos es del $30 \%$ al $60 \%{ }^{(12)}$.

Hay una relación, aunque relativamente débil, de ciertos alelos del HLA, como el DR3. El gen de la tiroglobulina se ha asociado con esta ETA. En un estudio de casos y controles se evaluaron 48 exones del gen de TG y se demostró que algunos polimorfismos de nucleótido único (SNP, por sus siglas en inglés) tendrían mayor riesgo con ETA (SNP de exón 10-12 y 33) en forma significativa $(p<0,01)$, y la combinación de ambos tendría una asociación aun mayor $(p<0,001)^{(13)}$.

La respuesta inicial autoinmunitaria por células T CD4+ parece generar una regulación al alta (upregulation) de la secreción de interferón gamma (IFN- $\gamma$ ), con un aumento de la expresión de las moléculas de clase HMC-II en los tirocitos. Este proceso posiblemente dispare la expansión de células $\mathrm{T}$ autorreactivas y da pie a la característica respuesta inflamatoria, con progresión de la enfermedad y generación de apoptosis, que, finalmente, lleva al hipotiroidismo. De igual forma, un fenómeno favorecedor parece ser la presencia de anticuerpos inhibidores de TSH circulantes. En relación con la lesión celular, la expresión del receptor CD95 y su ligando en el tejido tiroideo es mucho mayor que en las contrapartes normales, así como otros factores efectores de apoptosis, como caspasas 3 y 8, Bax, Bak y Fas/CD95 (3). En el proceso secundario, se han observado respuestas $\mathrm{CD} 4+\mathrm{y} \mathrm{CD} 8+$, con predominancia de CD4+. Por tal motivo, hay un incremento en la expresión de células $\mathrm{T}$ y sus marcadores como el HLA-DR, con citocinas como la interleucina 2 (IL-2), el IFN- $\gamma$, el factor de necrosis tumoral (FNT) y las IL-4, 6, 2, 10, 12, 13 y 15. Al presentarse actividad de la MHC-II, las expresiones de ICAM-1, LFA-3 y MHC-I por los tirocitos se incrementan por la IL-1, el FNT y el
IFN- $\gamma$, lo cual aumenta la capacidad citotóxica de las células T mediante la lisis. El ataque del complemento por la vía clásica o alternativa altera la función metabólica de la tiroides y las lleva a secretar IL-1, IL-6, especies reactivas de oxígeno y prostaglandinas, que incrementan el proceso autoinmunitario ${ }^{(3,4)}$.

\section{Infecciones}

En general, no se puede considerar un efecto de infección directa, aunque se ha demostrado la inducción en forma experimental en modelos animales.

Se han descrito casos de tiroiditis linfocítica tras inmunizaciones para estreptococo del grupo A y también se ha asociado a Yersinia enterocolítica. Se han reportado con frecuencia anticuerpos que pueden reaccionar con las proteínas del Western Blot para VIH-1, con datos que sugieren que los pacientes con infección por retrovirus no VIH producen anticuerpos para proteínas virales, con reactividad cruzada con proteínas VIH. De igual forma, se han descrito partículas de tipo viral, aunque su significancia clínica no es clara ${ }^{(4)}$.

En algunos estudios en humanos, se ha llevado a cabo la identificación virológica de la presencia directa de virus o sus componentes para retrovirus (HFV) y parotiditis en la tiroiditis subaguda, retrovirus (virus linfotrópico humano de células T tipo 1, HTLV-1, HFV, VIH y virus simiano 40, SV40) en Graves, y para HTLV-1, enterovirus, rubéola, parotiditis, HSV, EBV y parvovirus en tiroiditis de Hashimoto, pero, nuevamente, no es claro su papel como factores etiológicos directos o simplemente como espectadores "inocentes" (10).

\section{SARS-CoV-2/COVID-19 y ETA}

La captación del SARS-CoV-2 aerosolizado lleva a la infección de las células que expresan los receptores blancos de la enzima convertidora de la angiotensina (ECA-2), tales como las células alveolares tipo 2 y otras no conocidas. Se altera la respuesta del IFN antiviral, lo que resulta en replicación viral no controlada. La migración de neutrófilos y monocitos macrófagos termina hiperproduciendo citocinas proinflamatorias hasta llegar a un cuadro de "tormenta de citocinas", inflamación a la que contribuye la activación específica de Th1/ Th17. A su vez, las células B y las células plasmáticas producen anticuerpos específicos contra el SARS-CoV-2, que intentan la neutralización viral.

Existen mecanismos potenciales de evasión inmunitaria que son compartidos por el SARS-CoV, el MERS-CoV y el SARSCoV-2, como la interferencia del sistema inmunitario innato ${ }^{(14)}$.

Tras la epidemia en el 2003 del SARS como enfermedad viral en Asia, se ha reportado la posibilidad de enfermedad tiroidea como factor asociado a la gravedad del compromiso respiratorio. Dada la frecuencia de presentación de la ETA y la extensión global del brote (en dos docenas de países en Suramérica y Europa y en Norteamérica), era esperable la posibilidad de coexistencia de ambas patologías ${ }^{(15)}$. 
Aunque algunos de esos reportes sugieren una posible relación etiológica, al revisar las descripciones de compromiso pulmonar grave e hipotiroidismo concomitante, no es posible establecer causalidad. Estos casos pudieran corresponder a cuadros de tiroiditis subagudas. Lo que sí parece ser consistente es la posibilidad de mejoría clínica tras el inicio de la corrección del hipotiroidismo en el contexto del deterioro de los pacientes ${ }^{(16)}$.

Desde el punto de vista histopatológico, una evaluación en necropsias de 5 casos de SARS mostró una destrucción del epitelio folicular y exfoliación de las células al folículo, con un remanente de células epiteliales en el andamiaje tisular tiroideo escaso o ausente. En algunas áreas, las células epiteliales foliculares formaban acúmulos sin coloide. En otras zonas, las células epiteliales presentaban folículos dilatados, algunos se encontraban colapsados o distorsionados y otros presentaban una configuración microfolicular. Las células parafoliculares no pudieron ser identificadas en la evaluación rutinaria. En 1 de los casos se presentaban cambios capilares en el tejido conectivo interfolicular y el epitelio folicular presentaba daño grave. Sin embargo, no se evidenció infiltración neutrofílica ni linfoide en el tejido conectivo. En las mujeres se evidenció, adicionalmente, fibrosis interfolicular.

En la evaluación específica de apoptosis se evidenció compromiso en el epitelio folicular y en las regiones interfoliculares de todos los pacientes. De igual forma, en la totalidad de las muestras de SARS, las células calcitonina positivas estaban ausentes completamente ${ }^{(17)}$.

Aunque se ha planteado un origen multicausal, incluido el uso de corticoides para dichos hallazgos, se considera la posibilidad de daño directo, pues 1 de los 5 pacientes no recibió este tipo de medicación y presentaba iguales cambios ${ }^{(17)}$. La gravedad es variable y hay inclusive datos de tormenta tiroidea desencadenada por H1N1 ${ }^{(18)}$. Sin embargo, no encontramos gran cantidad de reportes de asociación entre tiroiditis y SARS-CoV o MERS, y menos aún datos de correlación entre SARS-CoV-2 y producción de ETA o tiroiditis.

En el caso particular de la COVID-19/SARS-CoV-2, no ha sido posible determinar una correlación con enfermedad tiroidea, aunque por la frecuencia de ambas patologías se esperaría una frecuente coexistencia. Por ahora, en la mayoría de las descripciones de características de los pacientes, no se menciona el compromiso tiroideo dentro de la comorbilidad presente. Recientemente se ha informado de un primer caso de tiroiditis subaguda relacionada con la infección por SARSCoV-2. En el artículo se describe a una mujer de 18 años, que consulta por fiebre, dolor de cuello irradiado a la mandíbula y palpitaciones que ocurrieron 15 días después de un hisopo orofaríngeo positivo para SARS-CoV-2. La COVID-19 había sido leve y la paciente se había recuperado por completo en pocos días. En los resultados del examen físico, la paciente presentó un aumento de la frecuencia cardíaca y una tiroides dolorosa y agrandada a la palpación. En los exámenes de laboratorio, la $\mathrm{T}_{4}$ libre y la $\mathrm{T}_{3}$ libre estaban elevadas, la TSH indetectable y marcadores inflamatorios y el recuento elevado de glóbulos blancos. Se detectaron áreas hipoecoicas bilaterales y difusas en el ultrasonido de cuello. Un mes antes, la función tiroidea y las imágenes eran normales. La respuesta a un ciclo corto de prednisona fue rápida, con desaparición de los síntomas en 1 semana, como es usual en la tiroiditis subaguda. La función tiroidea y los marcadores inflamatorios se normalizaron en 40 días. Los autores concluyeron que se trataba de tiroiditis subaguda posterior a la COVID-19 ${ }^{(19)}$

Otros factores de riesgo siguen presentando mayor relevancia para el riesgo de complicaciones en el caso de la infección por coronavirus reciente, como la hipertensión arterial, la diabetes y la obesidad. En una serie de casos en una población de pacientes hospitalizados en Estados Unidos (que incluyó 99 condados en 14 estados, con 1482 pacientes con infección confirmada por laboratorio en marzo de 2020), 180 (12,1\%) presentaban condiciones médicas asociadas y, de estos, el 89,3\% presentaba más de 1 comorbilidad. En cuanto a los 60 casos con enfermedades crónicas metabólicas, 13 tenían disfunción tiroidea aislada y 2 presentaban diabetes y disfunción tiroidea ${ }^{(20)}$.

En una reciente publicación de JAMA, no se reporta la presencia de enfermedad tiroidea en ninguno de los 5700 pacientes hospitalizados por COVID-19 en Nueva York, ni como consecuencia de la infección ${ }^{(21) .}$

Por otro lado, en un centro hospitalario en Wuhan, China, con confirmación por laboratorio de 140 pacientes, se reportó una frecuencia de enfermedad tiroidea en solo el 3,6\% de los casos $(n=5)^{(22)}$.

En el caso colombiano, existe información limitada, por no contarse con el reporte de comorbilidad en el total de infectados, y solo se dispone de algunos datos de pacientes fallecidos. En el análisis de los 100 primeros casos de mortalidad, 8 presentaban alteraciones tiroideas, reportados como hipotiroidismo (7) e hipertiroidismo (1) ${ }^{(23)}$.

Por lo anterior, al momento actual no es posible definir una relación entre la COVID-19 y la disfunción tiroidea más allá de la coexistencia y asociación, no causal, ni para el proceso de infección, ni para la comorbilidad o las complicaciones.

En cuanto a la correlación clínica, en 2003, en 48 pacientes con SARS se evidenció que los niveles de $\mathrm{T}_{3}, \mathrm{~T}_{4}$ y TSH fueron significativamente menores que en los controles, particularmente los valores de $\mathrm{T}_{3}{ }^{(24)}$.

Desde el punto de vista fisiológico, sin embargo, la reducción de los valores de TSH reportados no puede explicarse por la destrucción del epitelio folicular, ya que los valores de $\mathrm{T}_{3}$ y $\mathrm{T}_{4}$ bajos llevarían al incremento de TSH. Por tal motivo, se ha planteado que, asociada a la alteración tiroidea, se presentaría una disfunción a nivel hipofisario. En otro reporte de 4 de 61 pacientes supervivientes al SARS $(6,7 \%)$ se evidenció hipotiroidismo, incluidos 3 pacientes con hipotiroidismo central y 
1 con compromiso primario asociado a anticuerpos antitiroideos positivos. En estos casos se ha documentado hipocortisolismo central $(39,3 \%)^{(10,24,25)}$. Se plantean como mecanismos hipofisitis causada por el SARS, afección directa del hipotálamo; o por adaptación a la enfermedad crítica. Este planteamiento se ha definido con la presencia de edema y degeneración neuronal e identificación de las secuencias genómicas virales en el hipotálamo y la corteza cerebral de pacientes con SARS, además de disfunción adaptativa por la activación prolongada del eje hipotalámico-hipofisario-suprarrenal (HHS) ${ }^{(17)}$. Aun así, los resultados de los estudios de necropsias no son todos concluyentes y otros autores no han logrado la demostración de contenido viral en el tejido tiroideo, pero sí en paratiroides, hipófisis, páncreas y suprarrenales ${ }^{(26)}$.

\section{Desarrollo de autoinmunidad tiroidea en vacunación}

Tampoco se ha documentado asociación de alteración tiroidea autoinmunitaria después de la vacunación para SARS o MERS. En un estudio de tratamiento para cáncer de próstata, colon y páncreas, los pacientes tratados con GVAX (vacuna hecha con un tipo de célula tumoral transfectada con GM-CSF) desarrollaron anticuerpos antitiroglobulina TgAb y no antiperoxidasa. Esta producción resultó independiente del cáncer o del tratamiento simultáneo con ipilimumab, pero detectado con ciertos tipos de pruebas, que reconocen un epítope diferente a la que reconoce la prueba comercial rutinariamente utilizada en la tiroiditis de Hashimoto. Dicha seroconversión se correlacionó con un aumento de la sobrevida ${ }^{(27)}$. Hasta ahora parece lógico pensar que las vacunas contra el SARS-CoV-2 y el SARS-CoV deberían tener el mismo delineamiento, debido a la gran similitud genética entre ellos. Se han identificado epítopes en SARS-CoV en las células T y B derivadas delas proteínas de la espiga $(\mathrm{S})$ y nucleocápside $(\mathrm{N})$, que son idénticas a las de SARS-CoV-2. Son un blanco inmunitario dado, que no se han encontrado mutaciones en las 120 secuencias de SARSCoV-2 identificadas hasta ahora ${ }^{(28)}$.

Muchas enfermedades como el SARS-CoV, por ejemplo, dejan la sensación de buscar una vacuna como la mejor medida para evitar nuevas epidemias. Esto tiene dificultades que inician desde la comprensión del mecanismo patogénico del organismo agresor. La reproducibilidad de los modelos animales en humanos no es perfecta, por lo que, generalmente, debe probarse en humanos para evaluar su efectividad, además de realizarse ensayos clínicos en zonas endémicas y en la pospandemia. Todo esto le dificulta a las vacunas poder ganar aprobación para su uso generalizado ${ }^{(29)}$.

Actualmente no hay vacuna ni tratamiento farmacológico con eficacia clínica probada contra el síndrome respiratorio grave por SARS-CoV-2. De los 332 registros de ensayos clínicos en marzo de 2020, más de la mitad estaban reclutando y el resto en preparación para reclutar pacientes, de los cuales, muy pocos en África y Suramérica. Es por esto por lo que la Organización Mundial de la Salud (OMS) lanzó el estudio SOLIDARITY, un estudio internacional de tratamientos potenciales en África y Suramérica.

En la Tabla 2 se muestra la similitud en las secuencias proteicas entre el SARS-CoV-2 y el SARS-CoV y MERS, además de la dificultad en conseguir una vacuna contra las tres enfermedades. Parecido al SARS-CoV, un dominio de unión al receptor de MERS-CoV en la unión con la Fc es una vacuna que muestra adecuada actividad neutralizante, que bloquea la unión de la proteína S a su receptor, el hDPP-4 (DPP-4 humano). Ya se han desarrollado ratones (transgénicos o por entrega de hDPP-4 mediada por adenovirus) que expresan hDPP-4 y muestran susceptibilidad a la infección por MERS-CoV ${ }^{(30)}$.

En cuanto a los estudios de medicamentos para el tratamiento, la compañía Vir tiene un estudio con anticuerpos monoclonales, que busca determinar si los anticuerpos monoclonales anticoronavirus (mAbs) se unen a y neutralizan al nCOV-2019. En forma similar, la compañía Regeneron está en pruebas en humanos con anticuerpos monoclonales contra el MERS y está en desarrollo de anticuerpos monoclonales contra el nCoV-2019.

En la línea de desarrollo global hay por lo menos 15 candidatos a vacunas, que emplean una diversidad de tecnologías, como ARN mensajero, nanopartículas basadas en ADN, partículas tipo virus sintéticas y modificadas. A excepción de aquellos estudios de la Coalition for Epidemic Preparedness Innovations (CEPI), la fase 1 no iniciará antes de 1 año. No se están realizando actualmente estudios en humanos para CoV-2019 (31).

La tecnología que usa células Vero (verda reno, en esperanto ["riñón verde"]) se convierte en una plataforma versátil de utilidad en el desarrollo de vacunas contra enfermedades virales emergentes. Dentro de esta gran variedad se encuentran el adenovirus, el virus del herpes y el ortomixovirus (H1N1), aunque también el SARS-CoV y el MERS ${ }^{(32) .}$

Tabla 2. Identidad de secuencias entre el SARS-CoV-2 y el SARS-CoV y MERS

\begin{tabular}{|c|c|c|c|c|}
\hline & Proteína S & Proteína N & Proteína M & Proteína E \\
\hline SARS-CoV & $76,0 \%$ & $90,6 \%$ & $90,1 \%$ & $94,7 \%$ \\
\hline MERS-CoV & $29,4 \%$ & $45,9 \%$ & $39,2 \%$ & $34,1 \%$ \\
\hline
\end{tabular}

Adaptada de la referencia 28. 


\section{SARS-CoV-2 y enfermedad tiroidea en enfermedad crítica}

El síndrome de la enfermedad no tiroidea (SENT), o como era llamado anteriormente: síndrome del eutiroideo enfermo

${ }^{(33)}$, es el nombre con el que se identifica a la alteración tiroidea en pacientes aguda y gravemente enfermos. Se acepta como una subregulación del eje hipotálamo-hipofisario-tiroideo (HHT), dado que cuanto mayor sea la alteración en el perfil bioquímico hormonal, mayor serán la gravedad y la mortalidad. La Figura 1 muestra la relación entre la gravedad de los cambios hormonales, que gradúa en leve, moderado o grave al SENT, y la mortalidad. Esta alteración puede entenderse como un mecanismo adaptativo o como una respuesta maladaptativa del organismo, en la que este no logra la producción hormonal requerida para el momento de la enfermedad crítica ${ }^{(33)}$.

Figura 1. Relación entre el perfil tiroideo y la mortalidad en el SENT. Adaptada de la referencia 39.

$\mathrm{T}_{3} \mathrm{~L}$ : hormona $\mathrm{T}_{3}$ libre; $\mathrm{T}_{3} \mathrm{r}$ : hormona $\mathrm{T}_{3}$ reversa; $\mathrm{T}_{3} \mathrm{~T}$ : hormona $\mathrm{T}_{3}$ total; $\mathrm{T}_{4} \mathrm{~L}$ : hormona $\mathrm{T}_{4}$ libre; $\mathrm{T}_{4} \mathrm{~T}$ : hormona $\mathrm{T}_{4}$ total; $\mathrm{TSH}$ : hormona estimulante de la tiroides.

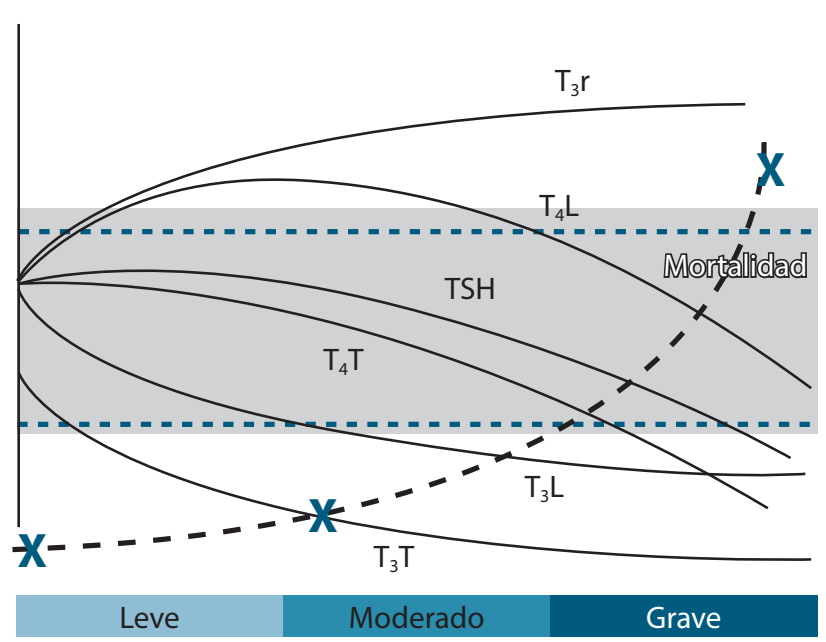

Se ha encontrado menor expresión de D1, sobreactivación en la deyonidasa 3 (D3), disregulación dela actividad periférica de $\mathrm{D} 2$, que lleva a reducción de $\mathrm{T}_{4} \mathrm{~L}$ y $\mathrm{T}_{3}$, con supresión de la liberación de TRH. Reducción de los transportadores MCT8 y MCT10, que altera el metabolismo normal de $\mathrm{T}_{3} \mathrm{y} \mathrm{T}_{4}, \mathrm{y}$ alteración de las proteínas transportadoras de hormonas tiroideas. También lleva a elevación de la $\mathrm{T}_{3}$ reversa como hormona inactiva $\left(\mathrm{T}_{3} \mathrm{r}\right){ }^{(34)}$.

Los pacientes en unidad de cuidados intensivos (UCI) frecuentemente reciben medicamentos que alteran la homeostasis del eje HHT, como, por ejemplo, los corticoides, los betabloqueadores, la amiodarona, la adrenalina, la noradrenalina, la dopamina, los opioides, la octreótida, la furosemida, la heparina, los salicilatos, la fenitoína, la carbamazepina metoclopramida, los medios de contraste yodados y, por ende, el perfil hormonal sanguíneo ${ }^{(35)}$.

Por último, la desnutrición y el desbalance entre el gasto y la ingesta calórica infrarregulan el eje HHT y empeoran la elevación de la $\mathrm{T}_{3}$ reversa circulante ${ }^{(36,37)}$.

Todo este perfil bioquímico se ha correlacionado con un aumento de la gravedad y la mortalidad; y, en general, se aceptan como marcador de gravedad el ingreso a UCI $(8,3 \%)$ y la mayor mortalidad a 30 días $(6,4 \%)$, con una diferencia significativa entre los supervivientes y los no supervivientes $(29,1$ versus $71,9 \% ; p<0,001$ ), aunque estos se consideraron en el contexto del síndrome de enfermedad no tiroidea ${ }^{(38)}$.

A medida que hay recuperación de la enfermedad de base, se produce una normalización en los niveles de hormona tiroidea y del eje HHT ${ }^{(39)}$. Las implicaciones de los valores bajos de $\mathrm{T}_{3}$ pudieran ir más allá del compromiso viral pulmonar o por SARS.

Si bien los reportes de compromiso pulmonar grave e insuficiencia ventilatoria durante la COVID-19 no mencionan lesión directa en la glándula tiroides, es apenas lógico que la función tiroidea se comprometa de la misma forma que en pacientes críticamente enfermos por otras causas ${ }^{(40)}$.

\section{Conclusiones}

Las enfermedades tiroideas son frecuentes en la población mundial, lo que hace probable la presentación de pacientes con ellas e infección por el SARS-CoV-2 o la COVID-19.

A la fecha, no hay demostración de que la COVID-19 sea más frecuente o grave en pacientes con ETA; en especial, no hay correlación con neumopatía grave en la COVID-19. Esto incluye al paciente crítico en UCI. En dado caso, es posible que se presente el cuadro clínico del síndrome de la enfermedad no tiroidea.

No existe evidencia que haga sospechar alteración tiroidea autoinmunitaria en casos de vacunación contra enfermedades similares a las producidas por el SARS-CoV-2.

Es importante que se estimule la continuación del tratamiento tiroideo recibido y un control adecuado durante la época de la pandemia y no se suspenda durante la hospitalización.

\section{Conflictos de interés}

Los autores declaran no tener conflicto de intereses para el presente artículo.

\section{Financiación}

Los autores declaran no haber tenido patrocinio ni financiación para el presente artículo. 


\section{Referencias}

1. Vanderpump MPJ, Tunbrldge WMG, French JM, Appleton D, Bates D, Clark $\mathrm{F}$, et al. The incidence of thyroid disorders in the community: a twenty-year follow-up of the Whickham Survey. Clin Endocrinol (Oxf). 1995;43(1):5568. doi: 10.1111/j.1365-2265.1995.tb01894.x.

2. Fröhlich E, Wahl R. Thyroid Autoimmunity: Role of Anti-thyroid Antibodies in Thyroid and Extra-Thyroidal Diseases. Front Immunol. 2017;8:521. doi: 10.3389/fimmu.2017.00521/full.

3. Iddah MA, Macharia BN. Autoimmune Thyroid Disorders. 2013:509764. doi: $10.1155 / 2013 / 509764$.

4. Tomer Y, Davies TF. Infection, Thyroid Disease, and Autoimmunity. Endocr Rev. 1993;14(1):107-20. doi: 10.1210/edrv-14-1-107.

5. Berger SA, Zonszein J, Villamena P, Mittman N. Infectious Diseases of the Thyroid Gland. Clin Infect Dis. 1983;5(1):108-22. doi: 10.1093/clinids/5.1.108.

6. Wang PW, Liu RT, Juo SHH, Wang ST, Hu YH, Hsieh CJ, et al. Cytotoxic T Lymphocyte-Associated Molecule-4 Polymorphism and Relapse of Graves' Hyperthyroidism after Antithyroid Withdrawal. J Clin Endocrinol Metab. 2004;89(1):169-73. doi: 10.1210/jc.2003-030854.

7. Martin A, Barbesino G, Davies TF. T-Cell Receptors and Autoimmune Thyroid Disease-Signposts for T-Cell-Antigen Driven Diseases. Int Rev Immunol. 1999;18(1-2):111-40. doi: 10.3109/08830189909043021.

8. Arata N, Ando T, Unger P, Davies TF. By-stander activation in autoimmune thyroiditis: Studies on experimental autoimmune thyroiditis in the GFP+ fluorescent mouse. Clin Immunol. 2006;121(1):108-17. doi: 10.1016/j. clim.2006.03.011.

9. Werner J, Gelderblom H. Isolation of foamy virus from patients with de Quervain thyroiditis. Lancet. 1979;2(8136):258-9. doi: 10.1016/s01406736(79)90275-7.

10. Desailloud R, Hober D. Viruses and thyroiditis: an update. Virol J. 2009;6(1):5. doi: 10.1186/1743-422X-6-5.

11. Ohsako N, Tamai H, Sudo T, Mukuta T, Tanaka H, Kuma K, et al. Clinical characteristics of subacute thyroiditis classified according to human leukocyte antigen typing. J Clin Endocrinol Metab. 1995;80(12):3653-6. doi: 10.1210/jcem.80.12.8530615.

12. Brix TH, Kyvik KO, Hegedüs L. A Population-Based Study of Chronic Autoimmune Hypothyroidism in Danish Twins. J Clin Endocrinol Metab. 2000;85(2):536-9. doi: 10.1210/jcem.85.2.6385.

13. Ban Y, Greenberg DA, Concepcion E, Skrabanek L, Villanueva R, Tomer Y. Amino acid substitutions in the thyroglobulin gene are associated with susceptibility to human and murine autoimmune thyroid disease. Proc Natl Acad Sci U S A. 2003;100(25):15119-24. doi: 10.1073/pnas.2434175100.

14. Prompetchara E, Ketloy C, Palaga T. Immune responses in COVID-19 and potential vaccines: Lessons learned from SARS and MERS epidemic. Asian Pac J Allergy Immunol. 2020;38(1):1-9. doi: 10.12932/AP-200220-0772.

15. Centro para el Control y la Prevención de Enfermedades. Información básica sobre el SRAS [Internet]. [citado 4 de mayo de 2020]. Disponible en: https://www.cdc.gov/sars/about/fs-sars-sp.html.

16. Chetambath R, Parengal J, Aslam M, Shivashankaran S. Severe pneumonia in a young female with a possible causal relationship to hypothyroidism -A case report and review of literature. Int J Med App Res. 2017;2(2):17-24. Disponible en: https://bit.ly/2BhobGI.

17. Wei L, Sun S, Xu C, Zhang J, Xu Y, Zhu H, et al. Pathology of the thyroid in severe acute respiratory syndrome. Hum Pathol. 2007;38(1):95-102. doi: 10.1016/j.humpath.2006.06.011.

18. Baharoon SA. H1N1 infection-induced thyroid storm. Ann Thorac Med. 2010;5(2):110-2. doi: 10.4103/1817-1737.62475.

19. Brancatella A, Ricci D, Viola N, Sgrò D, Santini F, Latrofa F. Subacute Thyroiditis After Sars-COV-2 Infection. J Clin Endocrinol Metab. 2020;105(7):dgaa276. doi: 10.1210/clinem/dgaa276.

20. Garg S, Kim L, Whitaker M, O'Halloran A, Cummings C, Holstein R, et al. Hospitalization Rates and Characteristics of Patients Hospitalized with Laboratory-Confirmed Coronavirus Disease 2019 - COVID-NET, 14 States, March 1-30, 2020. MMWR Morb Mortal Wkly Rep. 2020;69(15):458-64. doi: $10.15585 / \mathrm{mmwr} . \mathrm{mm} 6915 \mathrm{e} 3$.

21. Richardson S, Hirsch J, Narasimhan M, Crawford JM, McGinn T, Davidson KW, et al. Presenting Characteristics, Comorbidities, and Outcomes Among 5700 Patients Hospitalized With COVID-19 in the New York City Area. JAMA. 2020;323(20):2052-9. doi: 10.1001/jama.2020.6775.
22. Zhang J, Dong X, Cao Y, Yuan Y, Yang Y, Yan Y, et al. Clinical characteristics of 140 patients infected with SARS-CoV-2 in Wuhan, China. Allergy 2020. doi: $10.1111 /$ all.14238.

23. Marín-Sánchez A. Características clínicas básicas en los primeros 100 casos de mortalidad Covid19-SARS2 en Colombia. Rev Panam Salud Pública Pan Am J Public Health. 2020.

24. Wang W, Ye YX, Yao H, et al. Evaluation and observation of serum thd parathyroid hormone in patients with severe acute respiratory syndrome [Internet]. CNKI. 2003. [citado 4 de mayo de 2020]. Disponible en: http:// en.cnki.com.cn/Article en/CJFDTotal-ZFLZ200304012.htm.

25. Leow MKS, Kwek DSK, Ng AWK, Ong KC, Kaw GJL, Lee LSU. Hypocortisolism in survivors of severe acute respiratory syndrome (SARS). Clin Endocrinol (Oxf). 2005;63(2):197-202. doi: 10.1111/j.1365-2265.2005.02325.x.

26. Ding Y, He L, Zhang Q, Huang Z, Che X, Hou J, et al. Organ distribution of severe acute respiratory syndrome (SARS) associated coronavirus (SARS$\mathrm{CoV}$ ) in SARS patients: implications for pathogenesis and virus transmission pathways. J Pathol. 2004;203(2):622-30. doi: 10.1002/path.1560.

27. De Remigis A, de Gruijl TD, Uram JN, Tzou SC, Iwama S, Talor MV, et al. Development of thyroglobulin antibodies after GVAX immunotherapy is associated with prolonged survival. Int J Cancer. 2015;136(1):127-37. doi 10.1002 /ijc.28973.

28. Ahmed SF, Quadeer AA, McKay MR. Preliminary Identification of Potential Vaccine Targets for the COVID-19 Coronavirus (SARS-CoV-2) Based on SARS-CoV Immunological Studies. Viruses. 2020;12(3):254. doi: 10.3390/ v12030254.

29. Yang Y, Peng F, Wang R, Guan K, Jiang T, Xu G, et al. The deadly coronaviruses: The 2003 SARS pandemic and the 2020 novel coronavirus epidemic in China. J Autoimmun. 2020;109:102434. doi: 10.1016/j.jaut.2020.102434.

30. Choi J, Kim MG, Oh YK, Kim YB. Progress of Middle East respiratory syndrome coronavirus vaccines: a patent review. Expert Opin Ther Pat. 2017;27(6):721-31. doi: 10.1080/13543776.2017.1281248.

31. Pang J, Wang MX, Ang IYH, Tan SHX, Lewis RF, Chen JI-P, et al. Potential Rapid Diagnostics, Vaccine and Therapeutics for 2019 Novel Coronavirus (2019-nCoV): A Systematic Review. J Clin Med. 2020;9(3):623. doi $10.3390 / \mathrm{jcm} 9030623$

32. Barrett PN, Terpening SJ, Snow D, Cobb RR, Kistner O. Vero cell technology for rapid development of inactivated whole virus vaccines for emerging viral diseases. Expert Rev Vaccines. 2017;16(9):883-94. doi: 10.1080/14760584.2017.1357471.

33. Boelen A, Kwakkel J, Fliers E. Beyond Low Plasma T3: Local Thyroid Hormone Metabolism during Inflammation and Infection. Endocr Rev. 2011;32(5):670-93. doi: 10.1210/er.2011-0007.

34. Peeters RP, van der Geyten S, Wouters PJ, Darras VM, van Toor H, Kaptein E, et al. Tissue Thyroid Hormone Levels in Critical Illness. J Clin Endocrinol Metab. 2005;90(12):6498-507. doi: 10.1210/jc.2005-1013.

35. Mebis L. The hypothalamus-pituitary-thyroid axis in critical illness. J Med. 2009;67(10):332-40.

36. Langouche L, Vander Perre S, Marques M, Boelen A, Wouters PJ, Casaer MP, et al. Impact of Early Nutrient Restriction During Critical Illness on the Nonthyroidal Illness Syndrome and Its Relation With Outcome: A Randomized, Controlled Clinical Study. J Clin Endocrinol Metab. 2013;98(3):100613. doi: $10.1210 /$ jc.2012-2809.

37. Schütz P, Bally M, Stanga Z, Keller U. Loss of appetite in acutely ill medica inpatients: physiological response or therapeutic target? Swiss Med Wkly. 2014;144:w13957. doi: 10.4414/smw.2014.13957.

38. Ringel MD. Management of Hypothyroidism and Hyperthyroidism in the Intensive Care Unit. Crit Care Clin. 2001;17(1):59-74. doi: 10.1016/s07490704(05)70152-4

39. Carrillo-Esper R, Peña-Pérez CA, Zárate-Vega V, Garcilazo-Reyes Y, Lee-Cervantes D, González-Noris PY. Disfunción tiroidea en el enfermo grave. Rev Invest Med Sur Mex. 2013;20(4):217-28.

40. Giamarellos-Bourboullis E, Netea M, Rovina N, Akinosoglou K, Antoniadou A, Antonakos N, et al. Complex Immune Dysregulation in COVID-19 Patients with Severe Respiratory Failure. Cell Host Microbe. 2020;27(6):992-1000. doi: 10.1016/j.chom.2020.04.009. 